Tautomerization of Methyldiazene to Formaldehyde-hydrazone in Ruthenium and Osmium Complexes

Gabriele Albertin, ${ }^{\star} \dagger$ Stefano Antoniutti,${ }^{\dagger}$ Alessia Bacchi,$\downarrow$ Fabiola De Marchi, ${ }^{\dagger}$ and Giancarlo Pelizzi

$\dagger$ Dipartimento di Chimica, Università Ca' Foscari di Venezia, Dorsoduro, 2137, 30123

Venezia, Italy

$\$$ Dipartimento di Chimica Generale ed Inorganica, Chimica Analitica, Chimica

Fisica,Università di Parma, Parco Area delle Scienze, 17/a, 43100 Parma, Italy

\title{
Crystallographic Analysis
}

Table S1. Crystal data and structure refinement for Compound 5·1/2 $\mathrm{CH}_{2} \mathrm{Cl}_{2}$.

Empirical formula

Formula weight

Temperature

Wavelength

Crystal system

Space group

Unit cell dimensions

Volume

Z

Density (calculated)

Absorption coefficient

$\mathrm{F}(000)$

Crystal size

Theta range for data collection

Index ranges

Reflections collected

Independent reflections

Completeness to theta $=21.57^{\circ}$

Refinement method

Data / restraints / parameters

Goodness-of-fit on $\mathrm{F}^{2}$

Final $\mathrm{R}$ indices [I $>2 \operatorname{sigma}(\mathrm{I})]$

$\mathrm{R}$ indices (all data)

Absolute structure parameter

Largest diff. peak and hole
$\mathrm{C}_{74.50} \mathrm{H}_{105} \mathrm{~B}_{2} \mathrm{Cl} \mathrm{N} \mathrm{O}_{13} \mathrm{P}_{4} \mathrm{Ru}$

1518.62

293(2) K

$0.71073 \AA$

orthorhombic

$P$ c a 21

$\mathrm{a}=22.239$ (1) $\AA$

$\mathrm{b}=19.041(1) \AA$

$\mathrm{c}=19.538(1) \AA$

8273.4(7) $\AA^{3}$

4

$1.219 \mathrm{Mg} / \mathrm{m}^{3}$

$0.356 \mathrm{~mm}^{-1}$

3204

$0.3 \times 0.1 \times 0.1 \mathrm{~mm}^{3}$

1.41 to $21.57^{\circ}$.

$-22<=\mathrm{h}<=22,-19<=\mathrm{k}<=19,-20<=\mathrm{l}<=20$

56361

$9553[\mathrm{R}($ int $)=0.0687]$

$99.8 \%$

Full-matrix least-squares on $\mathrm{F}^{2}$

9553 / 6 / 824

0.957

$\mathrm{R} 1=0.0465, \mathrm{wR} 2=0.1137$

$\mathrm{R} 1=0.0702, \mathrm{wR} 2=0.1216$

$-0.04(4)$

0.629 and -0.302 e. $\AA^{-3}$ 


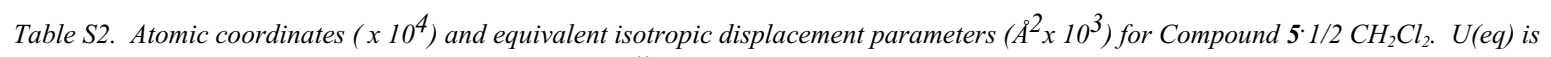
defined as one third of the trace of the orthogonalized $U^{i j}$ tensor.

\begin{tabular}{|c|c|c|c|c|}
\hline & $\mathrm{x}$ & $\mathrm{y}$ & z & $\mathrm{U}(\mathrm{eq})$ \\
\hline $\mathrm{Ru}$ & $4609(1)$ & $2140(1)$ & $2645(1)$ & $49(1)$ \\
\hline $\mathrm{N}(1)$ & $3699(3)$ & $1804(3)$ & 2989(4) & $63(2)$ \\
\hline $\mathrm{N}(2)$ & $3597(3)$ & $1057(4)$ & 2888(4) & $83(2)$ \\
\hline $\mathrm{O}(1)$ & $4408(3)$ & $1776(3)$ & $4203(3)$ & $79(2)$ \\
\hline $\mathrm{O}(2)$ & $5193(3)$ & $927(3)$ & $3741(3)$ & $89(2)$ \\
\hline $\mathrm{O}(3)$ & $5498(3)$ & $2107(3)$ & $4017(3)$ & $80(2)$ \\
\hline $\mathrm{O}(4)$ & $4277(4)$ & $2030(4)$ & $1044(4)$ & $125(3)$ \\
\hline $\mathrm{O}(5)$ & $3442(2)$ & $2557(3)$ & $1769(3)$ & $94(2)$ \\
\hline $\mathrm{O}(6)$ & $4219(3)$ & $3288(4)$ & $1355(4)$ & $116(3)$ \\
\hline $\mathrm{O}(7)$ & $4596(2)$ & $3877(2)$ & $2715(4)$ & 81(2) \\
\hline $\mathrm{O}(8)$ & $4632(3)$ & $3435(3)$ & $3886(3)$ & $85(2)$ \\
\hline $\mathrm{O}(9)$ & $3713(2)$ & $3254(3)$ & $3264(3)$ & $85(2)$ \\
\hline $\mathrm{O}(10)$ & $5813(2)$ & $3106(2)$ & $2596(4)$ & $86(2)$ \\
\hline $\mathrm{O}(11)$ & $5666(3)$ & $2456(3)$ & $1538(3)$ & $90(2)$ \\
\hline $\mathrm{O}(12)$ & $6001(2)$ & 1816(3) & $2567(4)$ & $90(2)$ \\
\hline $\mathrm{O}(13)$ & $4799(3)$ & $714(3)$ & 1977(3) & $86(2)$ \\
\hline $\mathrm{P}(1)$ & 4957(1) & $1702(1)$ & $3694(1)$ & $63(1)$ \\
\hline $\mathrm{P}(2)$ & $4139(1)$ & $2547(1)$ & $1646(1)$ & $64(1)$ \\
\hline $\mathrm{P}(3)$ & $4418(1)$ & $3245(1)$ & $3158(1)$ & $59(1)$ \\
\hline $\mathrm{P}(4)$ & $5562(1)$ & $2400(1)$ & $2312(1)$ & $59(1)$ \\
\hline$C(1)$ & $4395(5)$ & $1597(6)$ & $4928(5)$ & 111(4) \\
\hline$C(2)$ & $4179(7)$ & $2184(6)$ & $5303(6)$ & $147(5)$ \\
\hline$C(3)$ & $4828(5)$ & $307(6)$ & $3757(7)$ & 127(4) \\
\hline$C(4)$ & $5128(6)$ & $-323(6)$ & $3716(11)$ & 221(9) \\
\hline$C(5)$ & $5978(5)$ & $1831(6)$ & $4423(6)$ & $107(3)$ \\
\hline$C(6)$ & $6244(8)$ & 2341(9) & $4855(9)$ & $226(10)$ \\
\hline$C(7)$ & 2982(4) & $2998(5)$ & $1458(5)$ & $100(3)$ \\
\hline $\mathrm{C}(8)$ & $2412(4)$ & $2649(5)$ & $1501(7)$ & 129(4) \\
\hline $\mathrm{C}(9)$ & $3900(7)$ & $1972(8)$ & 411(7) & $178(7)$ \\
\hline$C(10)$ & $4261(7)$ & $1683(9)$ & $-93(7)$ & $178(6)$ \\
\hline$C(11)$ & $4685(6)$ & $3629(7)$ & $1085(8)$ & $137(5)$ \\
\hline$C(12)$ & $4590(6)$ & $4326(6)$ & $795(6)$ & $141(5)$ \\
\hline C(13) & $4460(5)$ & $4614(4)$ & $2799(7)$ & 128(4) \\
\hline C(14) & $4945(5)$ & $5071(5)$ & $2573(7)$ & 129(4) \\
\hline$C(15)$ & $5145(5)$ & $3749(6)$ & $4089(6)$ & 114(4) \\
\hline$C(16)$ & $5162(5)$ & $4010(7)$ & $4777(6)$ & 134(4) \\
\hline$C(17)$ & $3360(4)$ & $3777(5)$ & $3645(5)$ & $87(3)$ \\
\hline $\mathrm{C}(18)$ & $2868(4)$ & $4008(5)$ & $3195(5)$ & $108(3)$ \\
\hline$C(19)$ & $6339(4)$ & $3474(5)$ & $2353(6)$ & $106(3)$ \\
\hline $\mathrm{C}(20)$ & $6728(6)$ & $3694(7)$ & $2899(6)$ & $155(5)$ \\
\hline $\mathrm{C}(21)$ & $6554(8)$ & $1618(10)$ & $2520(9)$ & $270(12)$ \\
\hline $\mathrm{C}(22)$ & $6803(4)$ & $996(5)$ & $2608(7)$ & $125(4)$ \\
\hline $\mathrm{C}(23)$ & $5833(9)$ & $2050(11)$ & $1069(8)$ & $238(11)$ \\
\hline$C(24)$ & $5966(6)$ & $2196(6)$ & $381(6)$ & $134(5)$ \\
\hline$C(25)$ & $4724(3)$ & $1246(5)$ & $2228(4)$ & $65(2)$ \\
\hline$C(26)$ & $3218(5)$ & $904(6)$ & $2464(7)$ & 104(4) \\
\hline$C(100)$ & $2059(3)$ & $9674(3)$ & $5415(3)$ & $70(2)$ \\
\hline C(101) & $2163(3)$ & $9244(3)$ & $5977(4)$ & 94(3) \\
\hline $\mathrm{C}(102)$ & $1687(4)$ & $9044(3)$ & $6395(3)$ & $104(3)$ \\
\hline C(103) & $1108(3)$ & $9274(4)$ & $6251(4)$ & $129(5)$ \\
\hline$C(104)$ & $1004(2)$ & $9705(4)$ & $5689(5)$ & $129(4)$ \\
\hline$C(105)$ & $1480(3)$ & $9905(3)$ & $5270(3)$ & 97(3) \\
\hline$C(106)$ & $2848(2)$ & $9267(3)$ & $4397(3)$ & $60(2)$ \\
\hline C(107) & $2665(2)$ & $8577(3)$ & $4499(3)$ & $72(2)$ \\
\hline C(108) & $2861(3)$ & $8052(2)$ & $4059(4)$ & 93(3) \\
\hline C(109) & $3240(3)$ & $8216(4)$ & $3516(3)$ & $102(3)$ \\
\hline$C(110)$ & $3423(3)$ & $8906(4)$ & $3414(3)$ & $109(3)$ \\
\hline$C(111)$ & $3226(3)$ & $9432(3)$ & $3854(3)$ & 83(3) \\
\hline $\mathrm{C}(112)$ & $3201(2)$ & $10110(3)$ & $5464(3)$ & $62(2)$ \\
\hline$C(113)$ & $3768(3)$ & $9812(3)$ & $5388(3)$ & $74(2)$ \\
\hline$C(114)$ & $4221(2)$ & 9971(3) & $5853(4)$ & $96(3)$ \\
\hline$C(115)$ & $4108(3)$ & $10428(4)$ & $6392(3)$ & 101(3) \\
\hline$C(116)$ & $3542(4)$ & $10726(3)$ & $6468(3)$ & 113(4) \\
\hline $\mathrm{C}(117)$ & $3088(3)$ & $10567(3)$ & $6003(3)$ & $84(3)$ \\
\hline$C(118)$ & $2465(3)$ & $10626(3)$ & $4438(3)$ & $68(2)$ \\
\hline C(119) & $2075(3)$ & $10525(3)$ & $3891(3)$ & $84(3)$ \\
\hline
\end{tabular}




$\begin{array}{lrrrr}\mathrm{C}(120) & 1913(3) & 11089(5) & 3479(3) & 106(3) \\ \mathrm{C}(121) & 2142(3) & 11754(4) & 3614(4) & 114(4) \\ \mathrm{C}(122) & 2532(3) & 11855(2) & 4161(4) & 97(3) \\ \mathrm{C}(123) & 2694(2) & 11291(4) & 4573(3) & 78(3) \\ \mathrm{C}(200) & 7651(3) & 4110(3) & 722(3) & 72(2) \\ \mathrm{C}(201) & 8165(3) & 4265(3) & 1097(3) & 87(3) \\ \mathrm{C}(202) & 8440(3) & 3746(4) & 1487(3) & 114(4) \\ \mathrm{C}(203) & 8200(3) & 3071(4) & 1501(3) & 105(3) \\ \mathrm{C}(204) & 7686(3) & 2917(2) & 1126(4) & 94(3) \\ \mathrm{C}(205) & 7411(2) & 3436(3) & 736(3) & 77(2) \\ \mathrm{C}(206) & 6802(3) & 4433(3) & -272(3) & 71(2) \\ \mathrm{C}(207) & 6218(3) & 4257(4) & -87(3) & 97(3) \\ \mathrm{C}(208) & 5835(2) & 3948(4) & -563(5) & 128(4) \\ \mathrm{C}(209) & 6037(4) & 3814(3) & -1223(4) & 114(4) \\ \mathrm{C}(210) & 6622(4) & 3989(4) & -1408(3) & 119(4) \\ \mathrm{C}(211) & 7004(3) & 4299(4) & -933(4) & 95(3) \\ \mathrm{C}(212) & 7799(2) & 5278(3) & -83(3) & 64(2) \\ \mathrm{C}(213) & 8253(3) & 4947(3) & -450(3) & 78(3) \\ \mathrm{C}(214) & 8666(2) & 5343(4) & -819(3) & 94(3) \\ \mathrm{C}(215) & 8625(3) & 6072(4) & -820(3) & 94(3) \\ \mathrm{C}(216) & 6172(3) & 6404(2) & -452(3) & 83(3) \\ \mathrm{C}(217) & 6007(3) & -84(3) & 67(2) \\ \mathrm{C}(218) & 6859(2) & 5225(3) & 909(3) & 69(2) \\ \mathrm{C}(219) & 7002(3) & 5146(3) & 1605(4) & 82(3) \\ \mathrm{C}(220) & 6664(3) & 5522(4) & 2079(2) & 94(3) \\ \mathrm{C}(221) & 6216(3) & 5976(3) & 1857(4) & 105(3) \\ \mathrm{C}(222) & 6104(2) & 6055(3) & 1161(4) & 98(3) \\ \mathrm{C}(223) & 6442(3) & 5679(3) & 687(3) & 94(3) \\ \mathrm{B}(1) & 2634(4) & 9918(5) & 4925(6) & 67(3) \\ \mathrm{B}(2) & 7285(4) & 4778(5) & 312(5) & 69(3) \\ \mathrm{Cl}(1) & 514(6) & 2338(7) & 3035(7) & 281(7) \\ \mathrm{Cl}(2) & -218(4) & 2551(7) & 2012(6) & 229(5) \\ \mathrm{C}(300) & -62(13) & 2068(13) & 2643(14) & 300(30) \\ & & & \end{array}$

Table S3. Bond lengths [A] and angles $\left[{ }^{\circ}\right]$ for Compound $51 / 2 \mathrm{CH}_{2} \mathrm{Cl}_{2}$.

\begin{tabular}{|c|c|c|c|c|c|}
\hline $\mathrm{Ru}-\mathrm{C}(25)$ & $1.904(10)$ & C(9)-C(10) & $1.384(17)$ & $C(200)-C(205)$ & 1.3900 \\
\hline $\mathrm{Ru}-\mathrm{N}(1)$ & $2.226(7)$ & $\mathrm{C}(11)-\mathrm{C}(12)$ & $1.457(16)$ & $\mathrm{C}(200)-\mathrm{B}(2)$ & $1.708(11)$ \\
\hline Ru-P(4) & $2.270(2)$ & $\mathrm{C}(13)-\mathrm{C}(14)$ & $1.454(11)$ & $C(201)-C(202)$ & 1.3900 \\
\hline $\mathrm{Ru}-\mathrm{P}(1)$ & $2.343(2)$ & $C(15)-C(16)$ & $1.435(14)$ & $C(202)-C(203)$ & 1.3900 \\
\hline $\mathrm{Ru}-\mathrm{P}(2)$ & $2.347(2)$ & $\mathrm{C}(17)-\mathrm{C}(18)$ & $1.470(11)$ & $\mathrm{C}(203)-\mathrm{C}(204)$ & 1.3900 \\
\hline $\mathrm{Ru}-\mathrm{P}(3)$ & $2.369(2)$ & $\mathrm{C}(19)-\mathrm{C}(20)$ & $1.435(13)$ & $C(204)-C(205)$ & 1.3900 \\
\hline $\mathrm{N}(1)-\mathrm{N}(2)$ & $1.454(9)$ & $\mathrm{C}(21)-\mathrm{C}(22)$ & $1.319(14)$ & $\mathrm{C}(206)-\mathrm{C}(207)$ & 1.3900 \\
\hline $\mathrm{N}(2)-\mathrm{C}(26)$ & $1.216(12)$ & $\mathrm{C}(23)-\mathrm{C}(24)$ & $1.404(17)$ & $\mathrm{C}(206)-\mathrm{C}(211)$ & 1.3900 \\
\hline $\mathrm{O}(1)-\mathrm{C}(1)$ & $1.458(11)$ & $\mathrm{C}(100)-\mathrm{C}(101)$ & 1.3900 & $\mathrm{C}(206)-\mathrm{B}(2)$ & $1.700(11)$ \\
\hline $\mathrm{O}(1)-\mathrm{P}(1)$ & $1.582(6)$ & $\mathrm{C}(100)-\mathrm{C}(105)$ & 1.3900 & C(207)-C(208) & 1.3900 \\
\hline $\mathrm{O}(2)-\mathrm{C}(3)$ & $1.432(11)$ & $\mathrm{C}(100)-\mathrm{B}(1)$ & $1.663(11)$ & C(208)-C(209) & 1.3900 \\
\hline $\mathrm{O}(2)-\mathrm{P}(1)$ & $1.570(6)$ & $\mathrm{C}(101)-\mathrm{C}(102)$ & 1.3900 & $\mathrm{C}(209)-\mathrm{C}(210)$ & 1.3900 \\
\hline $\mathrm{O}(3)-\mathrm{C}(5)$ & $1.429(11)$ & $\mathrm{C}(102)-\mathrm{C}(103)$ & 1.3900 & $C(210)-C(211)$ & 1.3900 \\
\hline $\mathrm{O}(3)-\mathrm{P}(1)$ & $1.562(6)$ & $C(103)-C(104)$ & 1.3900 & C(212)-C(213) & 1.3900 \\
\hline $\mathrm{O}(4)-\mathrm{C}(9)$ & $1.498(14)$ & $\mathrm{C}(104)-\mathrm{C}(105)$ & 1.3900 & $\mathrm{C}(212)-\mathrm{C}(217)$ & 1.3900 \\
\hline $\mathrm{O}(4)-\mathrm{P}(2)$ & $1.563(7)$ & $\mathrm{C}(106)-\mathrm{C}(107)$ & 1.3900 & $\mathrm{C}(212)-\mathrm{B}(2)$ & $1.677(10)$ \\
\hline $\mathrm{O}(5)-\mathrm{C}(7)$ & $1.455(9)$ & $\mathrm{C}(106)-\mathrm{C}(111)$ & 1.3900 & $\mathrm{C}(213)-\mathrm{C}(214)$ & 1.3900 \\
\hline $\mathrm{O}(5)-\mathrm{P}(2)$ & $1.569(6)$ & $\mathrm{C}(106)-\mathrm{B}(1)$ & $1.680(10)$ & $C(214)-C(215)$ & 1.3900 \\
\hline $\mathrm{O}(6)-\mathrm{C}(11)$ & $1.332(13)$ & $\mathrm{C}(107)-\mathrm{C}(108)$ & 1.3900 & $C(215)-C(216)$ & 1.3900 \\
\hline $\mathrm{O}(6)-\mathrm{P}(2)$ & $1.531(7)$ & $\mathrm{C}(108)-\mathrm{C}(109)$ & 1.3900 & $C(216)-C(217)$ & 1.3900 \\
\hline $\mathrm{O}(7)-\mathrm{C}(13)$ & $1.446(9)$ & $\mathrm{C}(109)-\mathrm{C}(110)$ & 1.3900 & $\mathrm{C}(218)-\mathrm{C}(219)$ & 1.3900 \\
\hline $\mathrm{O}(7)-\mathrm{P}(3)$ & $1.534(6)$ & $\mathrm{C}(110)-\mathrm{C}(111)$ & 1.3900 & $\mathrm{C}(218)-\mathrm{C}(223)$ & 1.3900 \\
\hline $\mathrm{O}(8)-\mathrm{C}(15)$ & $1.346(11)$ & $\mathrm{C}(112)-\mathrm{C}(113)$ & 1.3900 & $\mathrm{C}(218)-\mathrm{B}(2)$ & $1.690(11)$ \\
\hline $\mathrm{O}(8)-\mathrm{P}(3)$ & $1.543(6)$ & $\mathrm{C}(112)-\mathrm{C}(117)$ & 1.3900 & C(219)-C(220) & 1.3900 \\
\hline $\mathrm{O}(9)-\mathrm{C}(17)$ & $1.470(9)$ & $\mathrm{C}(112)-\mathrm{B}(1)$ & $1.682(11)$ & $C(220)-C(221)$ & 1.3900 \\
\hline $\mathrm{O}(9)-\mathrm{P}(3)$ & $1.581(5)$ & $\mathrm{C}(113)-\mathrm{C}(114)$ & 1.3900 & $\mathrm{C}(221)-\mathrm{C}(222)$ & 1.3900 \\
\hline $\mathrm{O}(10)-\mathrm{C}(19)$ & $1.444(9)$ & $\mathrm{C}(114)-\mathrm{C}(115)$ & 1.3900 & $\mathrm{C}(222)-\mathrm{C}(223)$ & 1.3900 \\
\hline $\mathrm{O}(10)-\mathrm{P}(4)$ & $1.557(5)$ & $C(115)-C(116)$ & 1.3900 & $\mathrm{Cl}(1)-\mathrm{C}(300)$ & $1.580(16)$ \\
\hline $\mathrm{O}(11)-\mathrm{C}(23)$ & $1.255(16)$ & $\mathrm{C}(116)-\mathrm{C}(117)$ & 1.3900 & $\mathrm{Cl}(2)-\mathrm{C}(300)$ & $1.577(16)$ \\
\hline $\mathrm{O}(11)-\mathrm{P}(4)$ & $1.534(7)$ & $\mathrm{C}(118)-\mathrm{C}(119)$ & 1.3900 & & \\
\hline $\mathrm{O}(12)-\mathrm{C}(21)$ & $1.291(13)$ & $\mathrm{C}(118)-\mathrm{C}(123)$ & 1.3900 & $\mathrm{C}(25)-\mathrm{Ru}-\mathrm{N}(1)$ & $89.7(3)$ \\
\hline $\mathrm{O}(12)-\mathrm{P}(4)$ & $1.561(6)$ & $\mathrm{C}(118)-\mathrm{B}(1)$ & $1.692(11)$ & $\mathrm{C}(25)-\mathrm{Ru}-\mathrm{P}(4)$ & $87.0(2)$ \\
\hline $\mathrm{O}(13)-\mathrm{C}(25)$ & $1.138(9)$ & $\mathrm{C}(119)-\mathrm{C}(120)$ & 1.3900 & N(1)-Ru-P(4) & $175.77(18)$ \\
\hline$C(1)-C(2)$ & $1.420(14)$ & $\mathrm{C}(120)-\mathrm{C}(121)$ & 1.3900 & C(25)-Ru-P(1) & $90.7(2)$ \\
\hline$C(3)-C(4)$ & $1.374(13)$ & $\mathrm{C}(121)-\mathrm{C}(122)$ & 1.3900 & N(1)-Ru-P(1) & $86.3(2)$ \\
\hline$C(5)-C(6)$ & $1.416(16)$ & $\mathrm{C}(122)-\mathrm{C}(123)$ & 1.3900 & $\mathrm{P}(4)-\mathrm{Ru}-\mathrm{P}(1)$ & $91.16(8)$ \\
\hline $\mathrm{C}(7)-\mathrm{C}(8)$ & $1.435(13)$ & $\mathrm{C}(200)-\mathrm{C}(201)$ & 1.3900 & C(25)-Ru-P(2) & $89.9(2)$ \\
\hline
\end{tabular}




$\begin{array}{lc}\mathrm{N}(1)-\mathrm{Ru}-\mathrm{P}(2) & 86.6(2) \\ \mathrm{P}(4)-\mathrm{Ru}-\mathrm{P}(2) & 96.01(8) \\ \mathrm{P}(1)-\mathrm{Ru}-\mathrm{P}(2) & 172.83(8) \\ \mathrm{C}(25)-\mathrm{Ru}-\mathrm{P}(3) & 177.4(2) \\ \mathrm{N}(1)-\mathrm{Ru}-\mathrm{P}(3) & 87.95(18) \\ \mathrm{P}(4)-\mathrm{Ru}-\mathrm{P}(3) & 95.44(7) \\ \mathrm{P}(1)-\mathrm{Ru}-\mathrm{P}(3) & 90.31(8) \\ \mathrm{P}(2)-\mathrm{Ru}-\mathrm{P}(3) & 88.76(8) \\ \mathrm{N}(2)-\mathrm{N}(1)-\mathrm{Ru} & 112.5(4) \\ \mathrm{C}(26)-\mathrm{N}(2)-\mathrm{N}(1) & 115.8(9) \\ \mathrm{C}(1)-\mathrm{O}(1)-\mathrm{P}(1) & 127.3(6) \\ \mathrm{C}(3)-\mathrm{O}(2)-\mathrm{P}(1) & 126.0(6) \\ \mathrm{C}(5)-\mathrm{O}(3)-\mathrm{P}(1) & 128.2(6) \\ \mathrm{C}(9)-\mathrm{O}(4)-\mathrm{P}(2) & 124.0(8) \\ \mathrm{C}(7)-\mathrm{O}(5)-\mathrm{P}(2) & 129.6(6) \\ \mathrm{C}(11)-\mathrm{O}(6)-\mathrm{P}(2) & 133.4(8) \\ \mathrm{C}(13)-\mathrm{O}(7)-\mathrm{P}(3) & 130.1(7) \\ \mathrm{C}(15)-\mathrm{O}(8)-\mathrm{P}(3) & 129.5(7) \\ \mathrm{C}(17)-\mathrm{O}(9)-\mathrm{P}(3) & 127.1(5) \\ \mathrm{C}(19)-\mathrm{O}(10)-\mathrm{P}(4) & 126.4(6) \\ \mathrm{C}(23)-\mathrm{O}(11)-\mathrm{P}(4) & 136.3(10) \\ \mathrm{C}(21)-\mathrm{O}(12)-\mathrm{P}(4) & 141.5(8) \\ \mathrm{O}(3)-\mathrm{P}(1)-\mathrm{O}(2) & 100.5(3) \\ \mathrm{O}(3)-\mathrm{P}(1)-\mathrm{O}(1) & 107.3(4) \\ \mathrm{O}(2)-\mathrm{P}(1)-\mathrm{O}(1) & 107.7(3) \\ \mathrm{O}(3)-\mathrm{P}(1)-\mathrm{Ru} & 115.6(2) \\ \mathrm{O}(2)-\mathrm{P}(1)-\mathrm{Ru} & 119.7(3) \\ \mathrm{O}(1)-\mathrm{P}(1)-\mathrm{Ru} & 105.3(2) \\ \mathrm{O}(6)-\mathrm{P}(2)-\mathrm{O}(4) & 106.1(5) \\ \mathrm{O}(6)-\mathrm{P}(2)-\mathrm{O}(5) & 108.4(4) \\ \mathrm{O}(4)-\mathrm{P}(2)-\mathrm{O}(5) & 124.2(3) \\ \mathrm{O}(6)-\mathrm{P}(2)-\mathrm{Ru} & 109.4(3) \\ \mathrm{O}(4)-\mathrm{P}(2)-\mathrm{Ru} & 108.5(2) \\ \mathrm{O}(5)-\mathrm{P}(2)-\mathrm{Ru} & 104.9(4) \\ \mathrm{O}(7)-\mathrm{P}(3)-\mathrm{O}(8) & 108.7(3) \\ \mathrm{O}(7)-\mathrm{P}(3)-\mathrm{O}(9) & 100.5(4) \\ \mathrm{O}(8)-\mathrm{P}(3)-\mathrm{O}(9) & \\ \mathrm{O}(7)-\mathrm{P}(3)-\mathrm{Ru} & \\ & \\ & \\ & \\ & \\ & \end{array}$

\begin{tabular}{|c|c|}
\hline $\mathrm{O}(8)-\mathrm{P}(3)-\mathrm{Ru}$ & $122.9(3)$ \\
\hline $\mathrm{O}(9)-\mathrm{P}(3)-\mathrm{Ru}$ & $104.0(2)$ \\
\hline $\mathrm{O}(11)-\mathrm{P}(4)-\mathrm{O}(10)$ & $103.7(4)$ \\
\hline $\mathrm{O}(11)-\mathrm{P}(4)-\mathrm{O}(12)$ & $105.6(4)$ \\
\hline $\mathrm{O}(10)-\mathrm{P}(4)-\mathrm{O}(12)$ & $106.0(3)$ \\
\hline $\mathrm{O}(11)-\mathrm{P}(4)-\mathrm{Ru}$ & $116.1(3)$ \\
\hline $\mathrm{O}(10)-\mathrm{P}(4)-\mathrm{Ru}$ & $114.9(2)$ \\
\hline $\mathrm{O}(12)-\mathrm{P}(4)-\mathrm{Ru}$ & $109.7(2)$ \\
\hline $\mathrm{C}(2)-\mathrm{C}(1)-\mathrm{O}(1)$ & $108.9(9)$ \\
\hline $\mathrm{C}(4)-\mathrm{C}(3)-\mathrm{O}(2)$ & $116.3(10)$ \\
\hline $\mathrm{C}(6)-\mathrm{C}(5)-\mathrm{O}(3)$ & $113.0(10)$ \\
\hline $\mathrm{C}(8)-\mathrm{C}(7)-\mathrm{O}(5)$ & $109.3(8)$ \\
\hline $\mathrm{C}(10)-\mathrm{C}(9)-\mathrm{O}(4)$ & $107.0(12)$ \\
\hline $\mathrm{O}(6)-\mathrm{C}(11)-\mathrm{C}(12)$ & $119.0(12)$ \\
\hline $\mathrm{O}(7)-\mathrm{C}(13)-\mathrm{C}(14)$ & $113.0(8)$ \\
\hline $\mathrm{O}(8)-\mathrm{C}(15)-\mathrm{C}(16)$ & $116.9(10)$ \\
\hline $\mathrm{C}(18)-\mathrm{C}(17)-\mathrm{O}(9)$ & $107.3(7)$ \\
\hline $\mathrm{C}(20)-\mathrm{C}(19)-\mathrm{O}(10)$ & $112.7(9)$ \\
\hline $\mathrm{O}(12)-\mathrm{C}(21)-\mathrm{C}(22)$ & $130.8(17)$ \\
\hline $\mathrm{O}(11)-\mathrm{C}(23)-\mathrm{C}(24)$ & $129.8(17$ \\
\hline $\mathrm{O}(13)-\mathrm{C}(25)-\mathrm{Ru}$ & $179.3(8)$ \\
\hline $\mathrm{C}(101)-\mathrm{C}(100)-\mathrm{C}(105)$ & 120.0 \\
\hline $\mathrm{C}(101)-\mathrm{C}(100)-\mathrm{B}(1)$ & $119.5(6)$ \\
\hline $\mathrm{C}(105)-\mathrm{C}(100)-\mathrm{B}(1)$ & $120.5(6)$ \\
\hline$C(100)-C(101)-C(102)$ & 120.0 \\
\hline $\mathrm{C}(103)-\mathrm{C}(102)-\mathrm{C}(101)$ & 120.0 \\
\hline$C(102)-C(103)-C(104)$ & 120.0 \\
\hline$C(105)-C(104)-C(103)$ & 120.0 \\
\hline $\mathrm{C}(104)-\mathrm{C}(105)-\mathrm{C}(100)$ & 120.0 \\
\hline$C(107)-C(106)-C(111)$ & 120.0 \\
\hline $\mathrm{C}(107)-\mathrm{C}(106)-\mathrm{B}(1)$ & $121.7(5)$ \\
\hline $\mathrm{C}(111)-\mathrm{C}(106)-\mathrm{B}(1)$ & $118.2(5)$ \\
\hline $\mathrm{C}(108)-\mathrm{C}(107)-\mathrm{C}(106)$ & 120.0 \\
\hline $\mathrm{C}(109)-\mathrm{C}(108)-\mathrm{C}(107)$ & 120.0 \\
\hline $\mathrm{C}(108)-\mathrm{C}(109)-\mathrm{C}(110)$ & 120.0 \\
\hline $\mathrm{C}(111)-\mathrm{C}(110)-\mathrm{C}(109)$ & 120.0 \\
\hline $\mathrm{C}(110)-\mathrm{C}(111)-\mathrm{C}(106)$ & 120.0 \\
\hline $\mathrm{C}(113)-\mathrm{C}(112)-\mathrm{C}(117)$ & 120.0 \\
\hline $\mathrm{C}(113)-\mathrm{C}(112)-\mathrm{B}(1)$ & $121.6(5)$ \\
\hline $\mathrm{C}(117)-\mathrm{C}(112)-\mathrm{B}(1)$ & $118.4(5)$ \\
\hline $\mathrm{C}(112)-\mathrm{C}(113)-\mathrm{C}(114)$ & 120.0 \\
\hline $\mathrm{C}(115)-\mathrm{C}(114)-\mathrm{C}(113)$ & 120.0 \\
\hline $\mathrm{C}(116)-\mathrm{C}(115)-\mathrm{C}(114)$ & 120.0 \\
\hline$C(117)-C(116)-C(115)$ & 120.0 \\
\hline $\mathrm{C}(116)-\mathrm{C}(117)-\mathrm{C}(112)$ & 120.0 \\
\hline $\mathrm{C}(119)-\mathrm{C}(118)-\mathrm{C}(123)$ & 120.0 \\
\hline $\mathrm{C}(119)-\mathrm{C}(118)-\mathrm{B}(1)$ & $117.5(6)$ \\
\hline $\mathrm{C}(123)-\mathrm{C}(118)-\mathrm{B}(1)$ & $122.5(6)$ \\
\hline $\mathrm{C}(118)-\mathrm{C}(119)-\mathrm{C}(120)$ & 120.0 \\
\hline $\mathrm{C}(121)-\mathrm{C}(120)-\mathrm{C}(119)$ & 120.0 \\
\hline $\mathrm{C}(120)-\mathrm{C}(121)-\mathrm{C}(122)$ & 120.0 \\
\hline
\end{tabular}

\begin{tabular}{|c|c|}
\hline$C(121)-C(122)-C(123)$ & 120.0 \\
\hline $\mathrm{C}(122)-\mathrm{C}(123)-\mathrm{C}(118)$ & 120.0 \\
\hline$C(201)-C(200)-C(205)$ & 120.0 \\
\hline $\mathrm{C}(201)-\mathrm{C}(200)-\mathrm{B}(2)$ & $118.7(5)$ \\
\hline $\mathrm{C}(205)-\mathrm{C}(200)-\mathrm{B}(2)$ & $121.0(5)$ \\
\hline$C(202)-C(201)-C(200)$ & 120.0 \\
\hline$C(203)-C(202)-C(201)$ & 120.0 \\
\hline$C(204)-C(203)-C(202)$ & 120.0 \\
\hline $\mathrm{C}(205)-\mathrm{C}(204)-\mathrm{C}(203)$ & 120.0 \\
\hline$C(204)-C(205)-C(200)$ & 120.0 \\
\hline $\mathrm{C}(207)-\mathrm{C}(206)-\mathrm{C}(211)$ & 120.0 \\
\hline $\mathrm{C}(207)-\mathrm{C}(206)-\mathrm{B}(2)$ & $120.6(6)$ \\
\hline $\mathrm{C}(211)-\mathrm{C}(206)-\mathrm{B}(2)$ & $119.4(6)$ \\
\hline $\mathrm{C}(208)-\mathrm{C}(207)-\mathrm{C}(206)$ & 120.0 \\
\hline$C(207)-C(208)-C(209)$ & 120.0 \\
\hline $\mathrm{C}(210)-\mathrm{C}(209)-\mathrm{C}(208)$ & 120.0 \\
\hline $\mathrm{C}(211)-\mathrm{C}(210)-\mathrm{C}(209)$ & 120.0 \\
\hline $\mathrm{C}(210)-\mathrm{C}(211)-\mathrm{C}(206)$ & 120.0 \\
\hline $\mathrm{C}(213)-\mathrm{C}(212)-\mathrm{C}(217)$ & 120.0 \\
\hline $\mathrm{C}(213)-\mathrm{C}(212)-\mathrm{B}(2)$ & $118.3(5)$ \\
\hline $\mathrm{C}(217)-\mathrm{C}(212)-\mathrm{B}(2)$ & $121.6(5)$ \\
\hline$C(212)-C(213)-C(214)$ & 120.0 \\
\hline $\mathrm{C}(215)-\mathrm{C}(214)-\mathrm{C}(213)$ & 120.0 \\
\hline$C(214)-C(215)-C(216)$ & 120.0 \\
\hline $\mathrm{C}(217)-\mathrm{C}(216)-\mathrm{C}(215)$ & 120.0 \\
\hline$C(216)-C(217)-C(212)$ & 120.0 \\
\hline $\mathrm{C}(219)-\mathrm{C}(218)-\mathrm{C}(223)$ & 120.0 \\
\hline $\mathrm{C}(219)-\mathrm{C}(218)-\mathrm{B}(2)$ & $121.9(6)$ \\
\hline $\mathrm{C}(223)-\mathrm{C}(218)-\mathrm{B}(2)$ & 118.1(6) \\
\hline $\mathrm{C}(220)-\mathrm{C}(219)-\mathrm{C}(218)$ & 120.0 \\
\hline$C(221)-C(220)-C(219)$ & 120.0 \\
\hline $\mathrm{C}(220)-\mathrm{C}(221)-\mathrm{C}(222)$ & 120.0 \\
\hline $\mathrm{C}(221)-\mathrm{C}(222)-\mathrm{C}(223)$ & 120.0 \\
\hline $\mathrm{C}(222)-\mathrm{C}(223)-\mathrm{C}(218)$ & 120.0 \\
\hline $\mathrm{C}(100)-\mathrm{B}(1)-\mathrm{C}(106)$ & $111.4(6)$ \\
\hline $\mathrm{C}(100)-\mathrm{B}(1)-\mathrm{C}(112)$ & $106.1(7)$ \\
\hline $\mathrm{C}(106)-\mathrm{B}(1)-\mathrm{C}(112)$ & $109.5(6)$ \\
\hline $\mathrm{C}(100)-\mathrm{B}(1)-\mathrm{C}(118)$ & $112.0(6)$ \\
\hline $\mathrm{C}(106)-\mathrm{B}(1)-\mathrm{C}(118)$ & $107.7(7)$ \\
\hline $\mathrm{C}(112)-\mathrm{B}(1)-\mathrm{C}(118)$ & $110.2(6)$ \\
\hline $\mathrm{C}(212)-\mathrm{B}(2)-\mathrm{C}(218)$ & $112.7(6)$ \\
\hline $\mathrm{C}(212)-\mathrm{B}(2)-\mathrm{C}(206)$ & $110.0(6)$ \\
\hline $\mathrm{C}(218)-\mathrm{B}(2)-\mathrm{C}(206)$ & $109.3(6)$ \\
\hline $\mathrm{C}(212)-\mathrm{B}(2)-\mathrm{C}(200)$ & $108.3(6)$ \\
\hline $\mathrm{C}(218)-\mathrm{B}(2)-\mathrm{C}(200)$ & $107.3(6)$ \\
\hline $\mathrm{C}(206)-\mathrm{B}(2)-\mathrm{C}(200)$ & $109.1(6)$ \\
\hline $\mathrm{Cl}(2)-\mathrm{C}(300)-\mathrm{Cl}(1)$ & $111.5(13)$ \\
\hline
\end{tabular}


Table S4. Anisotropic displacement parameters $\left(\AA^{2} \times 10^{3}\right)$ for Compound $5 \cdot 1 / 2 \mathrm{CH}_{2} \mathrm{Cl}_{2}$. The anisotropic displacement factor exponent takes the form: $-2 \pi^{2}\left[h^{2} a^{* 2} U^{11}+\ldots+2 h k a^{*} b^{*} U^{12}\right]$

\begin{tabular}{|c|c|c|c|c|c|c|}
\hline & $\mathrm{U}^{11}$ & $\mathrm{U}^{22}$ & $\mathrm{U}^{33}$ & $\mathrm{U}^{23}$ & $\mathrm{U}^{13}$ & $\mathrm{U}^{12}$ \\
\hline $\mathrm{Ru}$ & $47(1)$ & $52(1)$ & $49(1)$ & $1(1)$ & $0(1)$ & $3(1)$ \\
\hline $\mathrm{N}(1)$ & $62(5)$ & $57(4)$ & $69(5)$ & $2(4)$ & $8(4)$ & $4(4)$ \\
\hline $\mathrm{N}(2)$ & $61(5)$ & $70(5)$ & $119(8)$ & $2(4)$ & $6(4)$ & $-11(4)$ \\
\hline $\mathrm{O}(1)$ & $90(4)$ & $100(4)$ & $47(4)$ & $18(3)$ & $3(3)$ & $1(4)$ \\
\hline $\mathrm{O}(2)$ & $107(5)$ & $68(4)$ & $93(4)$ & $16(3)$ & $0(4)$ & $18(4)$ \\
\hline $\mathrm{O}(3)$ & $87(4)$ & $87(4)$ & $67(4)$ & $7(3)$ & $-31(3)$ & $-2(3)$ \\
\hline $\mathrm{O}(4)$ & 141(7) & $162(7)$ & $72(5)$ & $-36(5)$ & $-38(5)$ & $39(5)$ \\
\hline $\mathrm{O}(5)$ & $65(4)$ & $133(5)$ & $83(4)$ & $38(4)$ & $-7(3)$ & $21(4)$ \\
\hline $\mathrm{O}(6)$ & $99(6)$ & $132(6)$ & $117(6)$ & $54(5)$ & $2(4)$ & $-8(5)$ \\
\hline $\mathrm{O}(7)$ & $109(4)$ & $64(3)$ & $70(4)$ & $3(4)$ & $4(4)$ & $1(3)$ \\
\hline $\mathrm{O}(8)$ & $107(5)$ & $69(4)$ & $77(4)$ & $-7(3)$ & $-12(4)$ & $-17(4)$ \\
\hline $\mathrm{O}(9)$ & $58(4)$ & $76(4)$ & $120(5)$ & $-34(4)$ & $14(3)$ & $16(3)$ \\
\hline $\mathrm{O}(10)$ & $68(3)$ & $77(3)$ & $114(5)$ & $-23(4)$ & $34(4)$ & $-26(3)$ \\
\hline $\mathrm{O}(11)$ & $122(5)$ & $91(4)$ & $56(4)$ & $2(3)$ & $28(4)$ & $24(4)$ \\
\hline $\mathrm{O}(12)$ & $58(3)$ & $124(4)$ & $87(4)$ & $15(5)$ & $16(4)$ & $42(3)$ \\
\hline $\mathrm{O}(13)$ & $94(5)$ & $66(4)$ & $98(5)$ & $-19(4)$ & $9(4)$ & $6(3)$ \\
\hline $\mathrm{P}(1)$ & $62(1)$ & $65(1)$ & $61(1)$ & $9(1)$ & $0(1)$ & $5(1)$ \\
\hline $\mathrm{P}(2)$ & $64(2)$ & $73(1)$ & $53(1)$ & $7(1)$ & $-2(1)$ & $9(1)$ \\
\hline $\mathrm{P}(3)$ & $63(2)$ & $57(1)$ & $57(1)$ & $0(1)$ & $5(1)$ & $0(1)$ \\
\hline$P(4)$ & $53(1)$ & $60(1)$ & $64(1)$ & $1(1)$ & $7(1)$ & $0(1)$ \\
\hline$C(1)$ & 131(9) & $140(10)$ & $62(7)$ & $16(7)$ & 1(6) & $12(8)$ \\
\hline $\mathrm{C}(2)$ & $234(16)$ & $124(10)$ & $84(8)$ & $-28(8)$ & $44(10)$ & $-33(10)$ \\
\hline$C(3)$ & $129(10)$ & $97(9)$ & $156(11)$ & $10(8)$ & $5(8)$ & $-12(8)$ \\
\hline C(4) & $170(13)$ & $66(8)$ & $430(30)$ & 11(13) & $-66(15)$ & $46(8)$ \\
\hline $\mathrm{C}(5)$ & $105(8)$ & $134(9)$ & $83(8)$ & $12(7)$ & $-19(7)$ & $7(7)$ \\
\hline$C(6)$ & 221(19) & 263(19) & 194(16) & $-102(15)$ & $-149(15)$ & $117(16)$ \\
\hline$C(7)$ & $89(8)$ & $117(8)$ & $96(7)$ & $5(6)$ & $-22(6)$ & $43(6)$ \\
\hline $\mathrm{C}(8)$ & $58(7)$ & $130(9)$ & 198(13) & $27(9)$ & $-24(7)$ & $14(6)$ \\
\hline C(9) & $222(17)$ & $209(16)$ & $103(11)$ & $-63(10)$ & $-62(11)$ & $74(13)$ \\
\hline $\mathrm{C}(10)$ & $225(17)$ & $230(17)$ & $78(9)$ & $-54(11)$ & $-42(10)$ & $-8(15)$ \\
\hline $\mathrm{C}(11)$ & $134(12)$ & $119(11)$ & $159(13)$ & $-27(10)$ & $22(10)$ & $-29(9)$ \\
\hline $\mathrm{C}(12)$ & 204(14) & $91(8)$ & $128(10)$ & $26(7)$ & $-31(9)$ & $36(8)$ \\
\hline$C(13)$ & $158(11)$ & $60(6)$ & $165(13)$ & $14(7)$ & $23(9)$ & $-22(6)$ \\
\hline $\mathrm{C}(14)$ & $152(9)$ & $86(6)$ & $149(10)$ & $-9(8)$ & $38(11)$ & $-32(6)$ \\
\hline$C(15)$ & $128(10)$ & $108(8)$ & 107(9) & $-6(7)$ & $-39(8)$ & $-11(7)$ \\
\hline$C(16)$ & $124(10)$ & $157(11)$ & $120(10)$ & $-54(8)$ & $0(8)$ & $-51(8)$ \\
\hline$C(17)$ & $66(6)$ & $100(7)$ & $95(7)$ & $-13(6)$ & $14(5)$ & $24(5)$ \\
\hline $\mathrm{C}(18)$ & $90(8)$ & 132(9) & $102(8)$ & $-10(7)$ & $3(6)$ & $41(6)$ \\
\hline C(19) & $95(8)$ & $96(7)$ & $128(9)$ & $9(6)$ & $-1(7)$ & $-33(6)$ \\
\hline $\mathrm{C}(20)$ & $134(11)$ & $187(12)$ & $144(13)$ & $-6(9)$ & $-38(9)$ & $-51(9)$ \\
\hline $\mathrm{C}(21)$ & 259(19) & $340(20)$ & 207(18) & $148(18)$ & $110(16)$ & $247(19)$ \\
\hline $\mathrm{C}(22)$ & $76(6)$ & $142(8)$ & $157(10)$ & $-24(10)$ & $-34(9)$ & $45(6)$ \\
\hline $\mathrm{C}(23)$ & $250(20)$ & $390(30)$ & $80(11)$ & $-6(14)$ & $6(12)$ & $170(20)$ \\
\hline$C(24)$ & $183(13)$ & $135(10)$ & $83(8)$ & $-18(7)$ & $62(8)$ & $-21(9)$ \\
\hline$C(25)$ & $43(5)$ & $85(6)$ & $66(5)$ & $11(5)$ & $2(4)$ & $-17(4)$ \\
\hline$C(26)$ & $72(7)$ & $93(8)$ & $147(13)$ & $-2(8)$ & $4(7)$ & $-6(7)$ \\
\hline$C(100)$ & $61(6)$ & $69(6)$ & $80(6)$ & $-12(5)$ & $0(5)$ & $8(5)$ \\
\hline $\mathrm{C}(101)$ & $96(8)$ & $94(8)$ & $91(8)$ & $7(6)$ & $18(7)$ & $0(6)$ \\
\hline$C(102)$ & 124(9) & $94(8)$ & $95(8)$ & $3(6)$ & $37(8)$ & $-5(7)$ \\
\hline C(103) & $97(10)$ & $126(10)$ & $165(13)$ & $-39(9)$ & $72(9)$ & $-22(8)$ \\
\hline C(104) & $86(9)$ & $144(11)$ & $156(12)$ & $11(9)$ & $24(8)$ & $1(7)$ \\
\hline$C(105)$ & $56(6)$ & $118(8)$ & 119(9) & $-7(7)$ & $7(6)$ & $15(6)$ \\
\hline$C(106)$ & $55(6)$ & $65(6)$ & $59(6)$ & $-4(5)$ & $-18(4)$ & $8(4)$ \\
\hline C(107) & $82(6)$ & $62(5)$ & $73(6)$ & $-4(5)$ & $-4(5)$ & $-3(5)$ \\
\hline C(108) & $104(8)$ & $69(6)$ & $106(8)$ & $-20(6)$ & $-16(7)$ & $15(5)$ \\
\hline C(109) & 103(8) & 113(9) & $90(8)$ & $-43(7)$ & $-16(6)$ & $47(7)$ \\
\hline $\mathrm{C}(110)$ & 116(9) & 121(9) & $88(8)$ & $-13(7)$ & $15(6)$ & $35(8)$ \\
\hline $\mathrm{C}(111)$ & $88(7)$ & $81(7)$ & $80(7)$ & $-10(6)$ & $12(6)$ & $3(5)$ \\
\hline$C(112)$ & $65(6)$ & $62(5)$ & $60(6)$ & $6(5)$ & $4(5)$ & $-6(4)$ \\
\hline $\mathrm{C}(113)$ & $57(6)$ & $75(6)$ & $90(7)$ & $12(5)$ & $-15(5)$ & $-8(5)$ \\
\hline $\mathrm{C}(114)$ & $93(8)$ & $85(7)$ & $110(8)$ & $32(6)$ & $-20(7)$ & $-17(6)$ \\
\hline $\mathrm{C}(115)$ & $90(8)$ & $110(9)$ & $102(9)$ & $37(7)$ & $-35(7)$ & $-27(7)$ \\
\hline$C(116)$ & $165(12)$ & $105(8)$ & $67(7)$ & $-3(6)$ & $-4(8)$ & $-41(9)$ \\
\hline$C(117)$ & $97(7)$ & $79(6)$ & $75(7)$ & $-12(5)$ & $-18(6)$ & $-12(5)$ \\
\hline $\mathrm{C}(118)$ & $78(6)$ & $77(6)$ & $49(5)$ & $3(4)$ & $14(4)$ & $21(5)$ \\
\hline C(119) & $98(7)$ & $96(7)$ & $59(6)$ & $1(6)$ & $-16(5)$ & $2(6)$ \\
\hline $\mathrm{C}(120)$ & 117(9) & 131(9) & $68(7)$ & $12(7)$ & $-8(6)$ & $33(8)$ \\
\hline $\mathrm{C}(121)$ & 115(9) & $124(10)$ & 103(9) & $48(8)$ & $28(7)$ & $67(8)$ \\
\hline $\mathrm{C}(122)$ & $90(7)$ & $80(7)$ & 121(9) & $14(7)$ & $31(7)$ & $12(6)$ \\
\hline $\mathrm{C}(123)$ & $78(6)$ & $59(5)$ & $98(7)$ & $6(5)$ & $12(5)$ & $4(5)$ \\
\hline$C(200)$ & $79(6)$ & $75(6)$ & $63(6)$ & $-1(5)$ & $2(5)$ & $4(5)$ \\
\hline$C(201)$ & $93(7)$ & $77(7)$ & $90(7)$ & $10(6)$ & $-24(6)$ & $-8(6)$ \\
\hline$C(202)$ & $94(8)$ & $144(11)$ & $105(9)$ & $31(8)$ & $-27(7)$ & $18(8)$ \\
\hline
\end{tabular}




$\begin{array}{lcccccc}\mathrm{C}(203) & 123(10) & 84(8) & 108(8) & 34(6) & 14(8) & 22(6) \\ \mathrm{C}(204) & 102(8) & 77(7) & 103(8) & 17(6) & 10(6) & -1(6) \\ \mathrm{C}(205) & 85(6) & 62(6) & 83(6) & 12(5) & 7(5) & 2(5) \\ \mathrm{C}(206) & 76(7) & 72(6) & 65(6) & 1(5) & -3(5) & 3(5) \\ \mathrm{C}(207) & 91(8) & 103(7) & 96(7) & -9(6) & -5(6) & -33(6) \\ \mathrm{C}(208) & 109(9) & 159(11) & 117(0) & -8(9) & -27(8) & -51(8) \\ \mathrm{C}(209) & 144(11) & 77(7) & 121(10) & -16(7) & -46(9) & -13(7) \\ \mathrm{C}(210) & 148(11) & 111(8) & 98(9) & -14(7) & -14(9) & -5(8) \\ \mathrm{C}(211) & 106(8) & 105(8) & 74(7) & -15(6) & -23(7) & -2(6) \\ \mathrm{C}(212) & 73(6) & 62(6) & 58(5) & 1(5) & -23(4) & 8(5) \\ \mathrm{C}(213) & 89(7) & 69(6) & 77(6) & 5(5) & 8(5) & 9(6) \\ \mathrm{C}(214) & 90(8) & 106(9) & 84(7) & 8(6) & -6(6) & 12(7) \\ \mathrm{C}(215) & 67(7) & 134(10) & 80(7) & 12(7) & -3(6) & -13(6) \\ \mathrm{C}(216) & 85(7) & 76(6) & 87(7) & 9(6) & -24(6) & -13(6) \\ \mathrm{C}(217) & 70(6) & 66(6) & 65(6) & -4(5) & -13(4) & -4(5) \\ \mathrm{C}(218) & 61(6) & 47(5) & 98(8) & 9(5) & 3(5) & -3(4) \\ \mathrm{C}(219) & 95(7) & 93(7) & 58(6) & 0(5) & -5(6) & -10(5) \\ \mathrm{C}(220) & 98(8) & 103(8) & 81(7) & -7(6) & -2(6) & -14(6) \\ \mathrm{C}(221) & 108(9) & 104(8) & 101(9) & -23(7) & 35(7) & -24(7) \\ \mathrm{C}(222) & 81(7) & 99(8) & 115(10) & -9(7) & 19(7) & 14(6) \\ \mathrm{C}(223) & 83(7) & 113(8) & 86(7) & -4(6) & 13(6) & 29(6) \\ \mathrm{B}(1) & 52(7) & 60(6) & 90(8) & -3(5) & 0(6) & -2(5) \\ \mathrm{B}(2) & 73(7) & 66(6) & 68(7) & 7(6) & -1(5) & -11(5) \\ \mathrm{Cl}(1) & 261(12) & 287(12) & 295(14) & 137(10) & -173(11) & -89(10) \\ \mathrm{Cl}(2) & 149(7) & 292(13) & 245(11) & -4(10) & -68(7) & 6(8) \\ \mathrm{C}(300) & 200(30) & 99(19) & 610(90) & 140(40) & 60(60) & -70(20) \\ & & & & & \end{array}$

Table S5. Hydrogen coordinates $\left(\times 10^{4}\right)$ and isotropic displacement parameters $\left(\AA^{2} \times 10^{3}\right)$ for Compound $5 \cdot 1 / 2 \mathrm{CH}_{2} \mathrm{Cl}_{2}$.

\begin{tabular}{|c|c|c|c|c|}
\hline & $\mathrm{x}$ & $\mathrm{y}$ & $\mathrm{Z}$ & $\mathrm{U}(\mathrm{eq})$ \\
\hline $\mathrm{H}(1 \mathrm{~A})$ & 4134 & 1196 & 5002 & 133 \\
\hline $\mathrm{H}(1 \mathrm{~B})$ & 4796 & 1474 & 5083 & 133 \\
\hline $\mathrm{H}(2 \mathrm{~A})$ & 4467 & 2558 & 5280 & 221 \\
\hline $\mathrm{H}(2 \mathrm{~B})$ & 4118 & 2051 & 5772 & 221 \\
\hline $\mathrm{H}(2 \mathrm{C})$ & 3804 & 2339 & 5110 & 221 \\
\hline $\mathrm{H}(3 \mathrm{~A})$ & 4597 & 311 & 4177 & 153 \\
\hline $\mathrm{H}(3 \mathrm{~B})$ & 4545 & 331 & 3380 & 153 \\
\hline $\mathrm{H}(4 \mathrm{~A})$ & 5445 & -287 & 3385 & 331 \\
\hline $\mathrm{H}(4 \mathrm{~B})$ & 4852 & -685 & 3580 & 331 \\
\hline $\mathrm{H}(4 \mathrm{C})$ & 5295 & -436 & 4155 & 331 \\
\hline $\mathrm{H}(5 \mathrm{~A})$ & 5825 & 1450 & 4702 & 129 \\
\hline $\mathrm{H}(5 \mathrm{~B})$ & 6284 & 1640 & 4122 & 129 \\
\hline $\mathrm{H}(6 \mathrm{~A})$ & 6476 & 2664 & 4583 & 339 \\
\hline $\mathrm{H}(6 \mathrm{~B})$ & 6503 & 2113 & 5179 & 339 \\
\hline $\mathrm{H}(6 \mathrm{C})$ & 5935 & 2594 & 5094 & 339 \\
\hline $\mathrm{H}(7 \mathrm{~A})$ & 2963 & 3445 & 1694 & 121 \\
\hline $\mathrm{H}(7 \mathrm{~B})$ & 3082 & 3086 & 982 & 121 \\
\hline $\mathrm{H}(8 \mathrm{~A})$ & 2430 & 2213 & 1256 & 193 \\
\hline $\mathrm{H}(8 \mathrm{~B})$ & 2106 & 2942 & 1304 & 193 \\
\hline $\mathrm{H}(8 \mathrm{C})$ & 2317 & 2559 & 1973 & 193 \\
\hline $\mathrm{H}(9 \mathrm{~A})$ & 3554 & 1674 & 494 & 214 \\
\hline $\mathrm{H}(9 \mathrm{~B})$ & 3759 & 2432 & 271 & 214 \\
\hline $\mathrm{H}(10 \mathrm{~A})$ & 4592 & 1994 & -186 & 267 \\
\hline $\mathrm{H}(10 \mathrm{~B})$ & 4030 & 1618 & -503 & 267 \\
\hline $\mathrm{H}(10 \mathrm{C})$ & 4413 & 1238 & 60 & 267 \\
\hline $\mathrm{H}(11 \mathrm{~A})$ & 4853 & 3335 & 728 & 165 \\
\hline $\mathrm{H}(11 \mathrm{~B})$ & 4989 & 3672 & 1438 & 165 \\
\hline $\mathrm{H}(12 \mathrm{~A})$ & 4289 & 4300 & 443 & 211 \\
\hline $\mathrm{H}(12 \mathrm{~B})$ & 4960 & 4495 & 603 & 211 \\
\hline $\mathrm{H}(12 \mathrm{C})$ & 4458 & 4640 & 1148 & 211 \\
\hline $\mathrm{H}(13 \mathrm{~A})$ & 4377 & 4707 & 3279 & 153 \\
\hline $\mathrm{H}(13 \mathrm{~B})$ & 4099 & 4725 & 2542 & 153 \\
\hline $\mathrm{H}(14 \mathrm{~A})$ & 5295 & 4988 & 2849 & 194 \\
\hline $\mathrm{H}(14 \mathrm{~B})$ & 4823 & 5552 & 2619 & 194 \\
\hline $\mathrm{H}(14 \mathrm{C})$ & 5037 & 4973 & 2103 & 194 \\
\hline $\mathrm{H}(15 \mathrm{~A})$ & 5223 & 4138 & 3782 & 137 \\
\hline $\mathrm{H}(15 \mathrm{~B})$ & 5471 & 3416 & 4034 & 137 \\
\hline $\mathrm{H}(16 \mathrm{~A})$ & 4803 & 4275 & 4868 & 200 \\
\hline $\mathrm{H}(16 \mathrm{~B})$ & 5507 & 4308 & 4834 & 200 \\
\hline $\mathrm{H}(16 \mathrm{C})$ & 5187 & 3623 & 5091 & 200 \\
\hline $\mathrm{H}(17 \mathrm{~A})$ & 3199 & 3570 & 4060 & 104 \\
\hline $\mathrm{H}(17 \mathrm{~B})$ & 3611 & 4173 & 3770 & 104 \\
\hline $\mathrm{H}(18 \mathrm{~A})$ & 3032 & 4262 & 2814 & 162 \\
\hline $\mathrm{H}(18 \mathrm{~B})$ & 2600 & 4307 & 3447 & 162 \\
\hline $\mathrm{H}(18 \mathrm{C})$ & 2652 & 3605 & 3031 & 162 \\
\hline $\mathrm{H}(19 \mathrm{~A})$ & 6561 & 3170 & 2046 & 128 \\
\hline H(19B) & 6212 & 3884 & 2096 & 128 \\
\hline
\end{tabular}




\begin{tabular}{|c|c|c|c|c|}
\hline $\mathrm{H}(20 \mathrm{~A})$ & 6513 & 4003 & 3200 & 232 \\
\hline $\mathrm{H}(20 \mathrm{~B})$ & 7069 & 3934 & 2712 & 232 \\
\hline $\mathrm{H}(20 \mathrm{C})$ & 6861 & 3289 & 3151 & 232 \\
\hline $\mathrm{H}(21 \mathrm{~A})$ & 6770 & 1925 & 2831 & 324 \\
\hline $\mathrm{H}(21 \mathrm{~B})$ & 6676 & 1759 & 2063 & 324 \\
\hline $\mathrm{H}(22 \mathrm{~A})$ & 6793 & 875 & 3085 & 187 \\
\hline $\mathrm{H}(22 \mathrm{~B})$ & 7213 & 1009 & 2454 & 187 \\
\hline $\mathrm{H}(22 \mathrm{C})$ & 6585 & 651 & 2350 & 187 \\
\hline $\mathrm{H}(23 \mathrm{~A})$ & 5526 & 1688 & 1052 & 286 \\
\hline $\mathrm{H}(23 \mathrm{~B})$ & 6191 & 1823 & 1245 & 286 \\
\hline $\mathrm{H}(24 \mathrm{~A})$ & 6140 & 2656 & 346 & 201 \\
\hline $\mathrm{H}(24 \mathrm{~B})$ & 5603 & 2178 & 115 & 201 \\
\hline $\mathrm{H}(24 \mathrm{C})$ & 6246 & 1854 & 211 & 201 \\
\hline $\mathrm{H}(101)$ & 2550 & 9089 & 6074 & 113 \\
\hline $\mathrm{H}(102)$ & 1756 & 8756 & 6771 & 125 \\
\hline $\mathrm{H}(103)$ & 789 & 9140 & 6531 & 155 \\
\hline $\mathrm{H}(104)$ & 617 & 9859 & 5592 & 154 \\
\hline $\mathrm{H}(105)$ & 1411 & 10193 & 4894 & 117 \\
\hline $\mathrm{H}(107)$ & 2412 & 8467 & 4862 & 87 \\
\hline H(108) & 2739 & 7590 & 4127 & 111 \\
\hline $\mathrm{H}(109)$ & 3371 & 7865 & 3222 & 122 \\
\hline $\mathrm{H}(110)$ & 3676 & 9017 & 3051 & 130 \\
\hline $\mathrm{H}(111)$ & 3349 & 9894 & 3786 & 99 \\
\hline $\mathrm{H}(113)$ & 3844 & 9506 & 5027 & 89 \\
\hline $\mathrm{H}(114)$ & 4601 & 9771 & 5802 & 115 \\
\hline $\mathrm{H}(115)$ & 4412 & 10534 & 6703 & 121 \\
\hline $\mathrm{H}(116)$ & 3466 & 11032 & 6829 & 135 \\
\hline $\mathrm{H}(117)$ & 2709 & 10767 & 6054 & 100 \\
\hline $\mathrm{H}(119)$ & 1922 & 10080 & 3801 & 101 \\
\hline $\mathrm{H}(120)$ & 1652 & 11022 & 3113 & 127 \\
\hline $\mathrm{H}(121)$ & 2034 & 12132 & 3338 & 137 \\
\hline $\mathrm{H}(122)$ & 2685 & 12300 & 4251 & 116 \\
\hline $\mathrm{H}(123)$ & 2955 & 11358 & 4939 & 94 \\
\hline $\mathrm{H}(201)$ & 8325 & 4716 & 1087 & 104 \\
\hline $\mathrm{H}(202)$ & 8784 & 3849 & 1738 & 137 \\
\hline $\mathrm{H}(203)$ & 8384 & 2724 & 1762 & 126 \\
\hline $\mathrm{H}(204)$ & 7526 & 2465 & 1136 & 113 \\
\hline $\mathrm{H}(205)$ & 7067 & 3332 & 485 & 92 \\
\hline $\mathrm{H}(207)$ & 6082 & 4347 & 355 & 116 \\
\hline $\mathrm{H}(208)$ & 5444 & 3831 & -439 & 154 \\
\hline $\mathrm{H}(209)$ & 5782 & 3607 & -1541 & 137 \\
\hline $\mathrm{H}(210)$ & 6757 & 3900 & -1850 & 143 \\
\hline $\mathrm{H}(211)$ & 7395 & 4416 & -1057 & 114 \\
\hline $\mathrm{H}(213)$ & 8280 & 4459 & -450 & 94 \\
\hline $\mathrm{H}(214)$ & 8969 & 5121 & -1064 & 112 \\
\hline $\mathrm{H}(215)$ & 8902 & 6337 & -1066 & 113 \\
\hline $\mathrm{H}(216)$ & 8145 & 6891 & -453 & 99 \\
\hline $\mathrm{H}(217)$ & 7455 & 6229 & 162 & 80 \\
\hline $\mathrm{H}(219)$ & 7302 & 4842 & 1754 & 98 \\
\hline $\mathrm{H}(220)$ & 6739 & 5469 & 2545 & 113 \\
\hline $\mathrm{H}(221)$ & 5990 & 6228 & 2174 & 126 \\
\hline $\mathrm{H}(222)$ & 5804 & 6359 & 1013 & 118 \\
\hline $\mathrm{H}(223)$ & 6367 & 5732 & 222 & 112 \\
\hline $\mathrm{H}(30 \mathrm{~A})$ & 6 & 1592 & 2485 & 363 \\
\hline $\mathrm{H}(30 \mathrm{~B})$ & -401 & 2060 & 2955 & 363 \\
\hline $\mathrm{H}(1 \mathrm{~N})$ & $3360(30)$ & $2070(40)$ & $2790(60)$ & $150(40)$ \\
\hline $\mathrm{H}(2 \mathrm{~N})$ & $3660(50)$ & $1910(60)$ & $3478(17)$ & $170(50)$ \\
\hline $\mathrm{H}(126)$ & $3200(40)$ & $347(13)$ & $2460(40)$ & $120(30)$ \\
\hline $\mathrm{H}(226)$ & $2980(30)$ & $1320(30)$ & $2230(40)$ & $100(30)$ \\
\hline
\end{tabular}




\section{Supporting Information}

Crystallographic Analysis

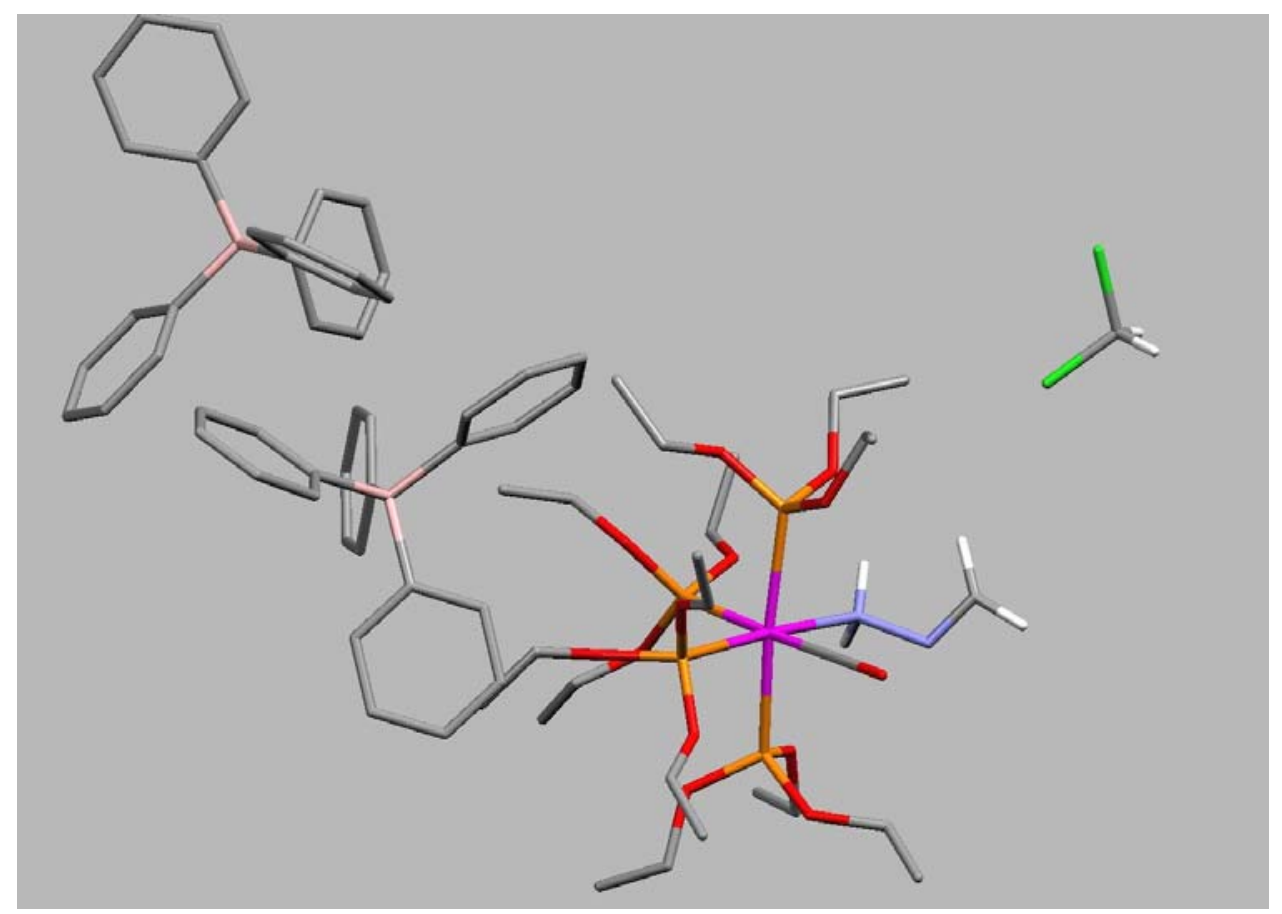

Figure S1. Asymmetric unit in the crystal structure of cis- $\left[\mathrm{Ru}(\mathrm{CO})\left(\mathrm{NH}_{2} \mathrm{~N}=\mathrm{CH}_{2}\right) \mathrm{P}_{4}\right]\left(\mathrm{BPh}_{4}\right) 2_{2} \cdot 0.5 \mathrm{CH}_{2} \mathrm{Cl}_{2}$. Hydrogens belonging to the anions and to ethoxy groups have been omitted. 